\title{
PSARP, a programmable signal and response processor
}

\section{H. v. DOORNE AND A. F. SANDERS, INSTITUTE FOR PERCEPTION RVO-TNO, Soesterberg, Netherlands}

Experiments on perceptual-motor skills often ask for complex application of standard devices. Combinations of the usual electric or electronic instruments such as signal generators, simple time counters and response recorders cannot always be used to form a sufficiently flexible system for a particular set of experiments. In general, an apparatus specially built for a specific experiment does not satisfy the requirements of related investigations and the same device is seldom used again. This paper gives an outline of the principles of a flexible and programmable system which meets the requirements of a considerable number of variables. Some first applications of the system are described.

Research on human skills and performance in man-machine system has given a strong impetus to the study of classical topics such as reaction time, short-term memory and prior entry. A basic difference between the present and the classical approach is that nowadays these topics are not treated as separate but are looked upon from the more general conceptions about man as an information processing system. This implies that more and more complex experiments are designed in which, say, RT processes, short-term storage, and selective attention are operating simultaneously. Examples can be found in work on the psychological refractory period (e.g., Bertelson, 1966), continuing memory (e.g., Sanders \& van Borselen, 1966) and dichotic listening (e.g., Broadbent \& Gregory, 1964). Mentioning these topics is only to sketch a broad outline of the subject, and the list can be easily extended.

Quite complicated apparatus is needed to meet the requirements of a more complex experiment. In principle there are sufficient technical possibilities to design the apparatus, mainly due to the rapid development of electronics. Development of special purpose apparatus for a particular experiment or even a group of experiments has serious disadvantages. However as the results of the experiments may easily ask for modifications of the apparatus, a growing need was felt for a more flexible system. Today, the easiest way to get such a system is: buy yourself a small on-line computer. However, in this paper a system, a Programmable Signal and Response Processor (PSARP, the $P$ is not pronounced) is described, which was needed and which could meet all requirements of psychological experiments at a time when small computers were not yet available. Moreover, PSARP might be used as a satellite system coupled to a computer in conditional response-signal feedback experiments.

\section{REQUIREMENTS}

In preliminary discussions about the equipment, the following requirements were formulated.

\section{Signals}

(1) A test session as a whole should be programmable. This includes the signals to be presented in a given trial, the length of the presentation time, the interval between trials and the number of trials.

(2) It should be possible to present several signals simul-

taneously, overlapping or successively. Presentation time of a particular signal and time intervals between particular signals should be variable with an accuracy of $1 \mathrm{msec}$.

(3) There should be a considerable set of alternative signals.

(4) Presentation should be possible at a paced or a self-paced rate.

(5) The system should be capable of controlling peripheral equipment with widely divergent properties (eg., lights, nixie tubes, acoustic signals, etc.).

Reactions

(1) The S's response and either $T$ (real time), $\Delta \mathrm{T}$ (time in terval between responses) or RT (reaction time) are to be recorded.

(2) Time intervals should be measured with an accuracy of $1 \mathrm{msec}$.

(3) Complex reactions (e.g., a sequence of reactions to one signal) should be possible.

(4) On line display as well as off line processing of the results should be possible.

With these requirements in mind a Programmable Signal and Response Processor (PSARP) was developed making use of digital techniques.

\section{Signal and Response Tapes}

\section{THE SYSTEM}

Data for the signals are fed into PSARP from a punched tape by means of a very fast optical reader. This signal tape contains data for the signal patterns, warning signals, and intervals between successive presentations, implying that a whole test session is preprogrammed. The signal tape is assembled by a PDP-7 digital computer, using a program which was specially written for PSARP. Running this program, a sequence of standard signal patterns is assembled and punched on paper tape, constituting the signal tape. The standard patterns are manually prepared in advance by means of a special purpose keyboard, controlling a tape punch.

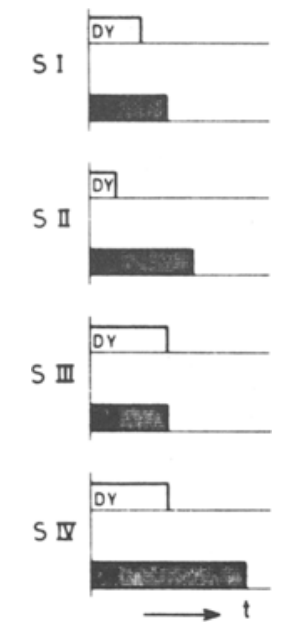

Fig. 1. Time values for the sets in a signal group.

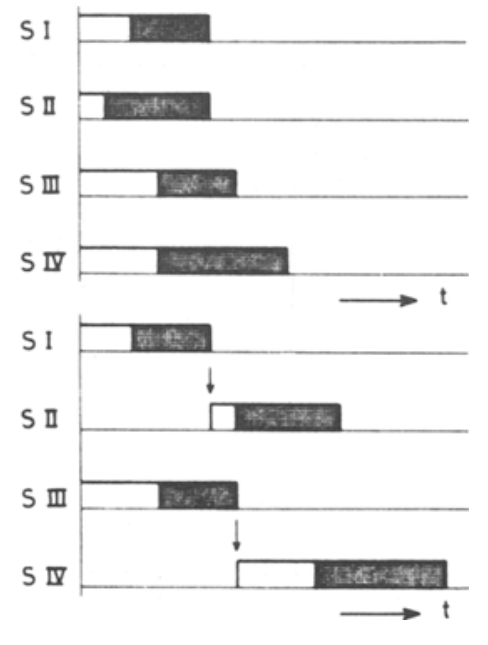

Fig. 2. Above: pattern for parallel actions. Below: pattern for parallel and serial actions. 


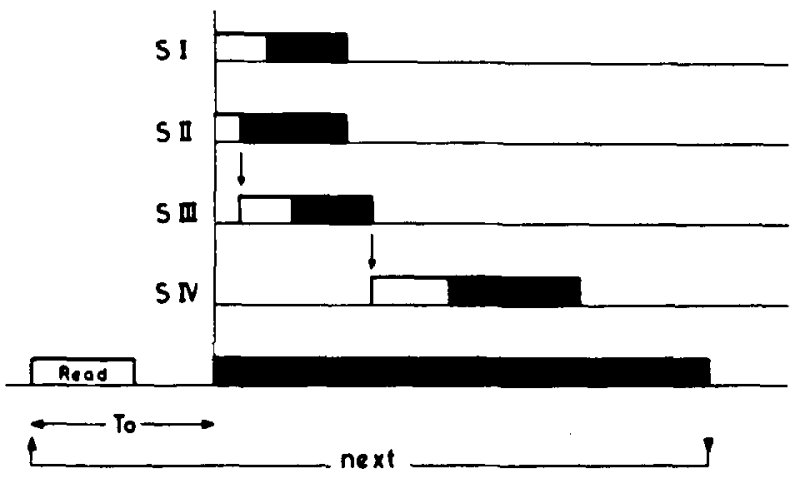

Fig. 3. Automatic pattern presentation

Responses and times together with appropriate signal pattern numbers can be printed by means of a digital printer and/or punched on paper tape. This tape may be used as data tape in computer programs for the analysis of results.

\section{Selection and Timing of Signals}

A maximum of four signals, each to be drawn from a set of eight, can be presented either simultaneously or overlapping in one cycle of the system. The signals are produced by actions of apparatus connected with the individual outputs of four Device Selectors. In each selector one device can be timed once in a cycle, so four devices can act simultaneously. They are in the on-state for a time interval called Duration, which follows after a time interval called Delay. This delay realizes the possibility of overlapping, signals. The devices connected with a selector constitute a set. The devices themselves are called Individuals. So there are four sets, having eight individuals each. Set numbers,. Individual numbers, and the values of Delay and Duration (Fig. 1) form a signal group. A group is read from the punched tape in each cycle. Device selectors and the preset counters of the four signal sets (SI, SII, SIII, and SIV, Fig. 6) are preset by means of the Input Data Control (Fig. 4). The stimulation process is started at a time $T_{0}$ af ter the total group information has been read in. The sequence in which the four sets start to count down their preset delay and duration time is programmable by the Matrix Switching Board, which is a part of the Timing Control (Fig. 4). On this board the instant at which the counters start can be chosen to fall at a fixed time $T_{0}$ after the beginning of the read cycle $\left(T_{0}=60 \mathrm{msec}\right.$ or $\left.100 \mathrm{msec}\right)$. Alternatively this can be made to coincide with instants at which other counters stop. So, complex timing can be achieved, while different timing patterns can be realized (Fig. 2) using only a single punched tape.

In the case of automatic presentation of signal patterns one additional value is read in within each group. This value presets a counter which determines the time interval between two successive signal patterns. In this time interval the signals are presented. This counter can be started in the same way as the delay and duration counters. As it stops a new cycle of the system is initialized and data for the next signal pattern will be read in. In this way the time space between successive signal patterns is programmable (Fig. 3). Because this counter determines the time for each cycle of the system, it is called the Cycle Time Counter (Fig. 4).

In order to have maximal flexibility, all counters can be pulsed independently with time base pulse signals, which for each counter can be chosen by switches. The internal time base is $1 \mathrm{msec}$, but derivations of 2,5 , and $10 \mathrm{msec}$ are also available. External standards can be used choosing the proper switch settings. In one setting which is called "coded," the time base for each signal pattern is defined by a time base code in the signal group read in. In this way the program tape itself determines the momentary time base for each signal pattern.

Fig. 4. Block diagram of sig. nal processing.

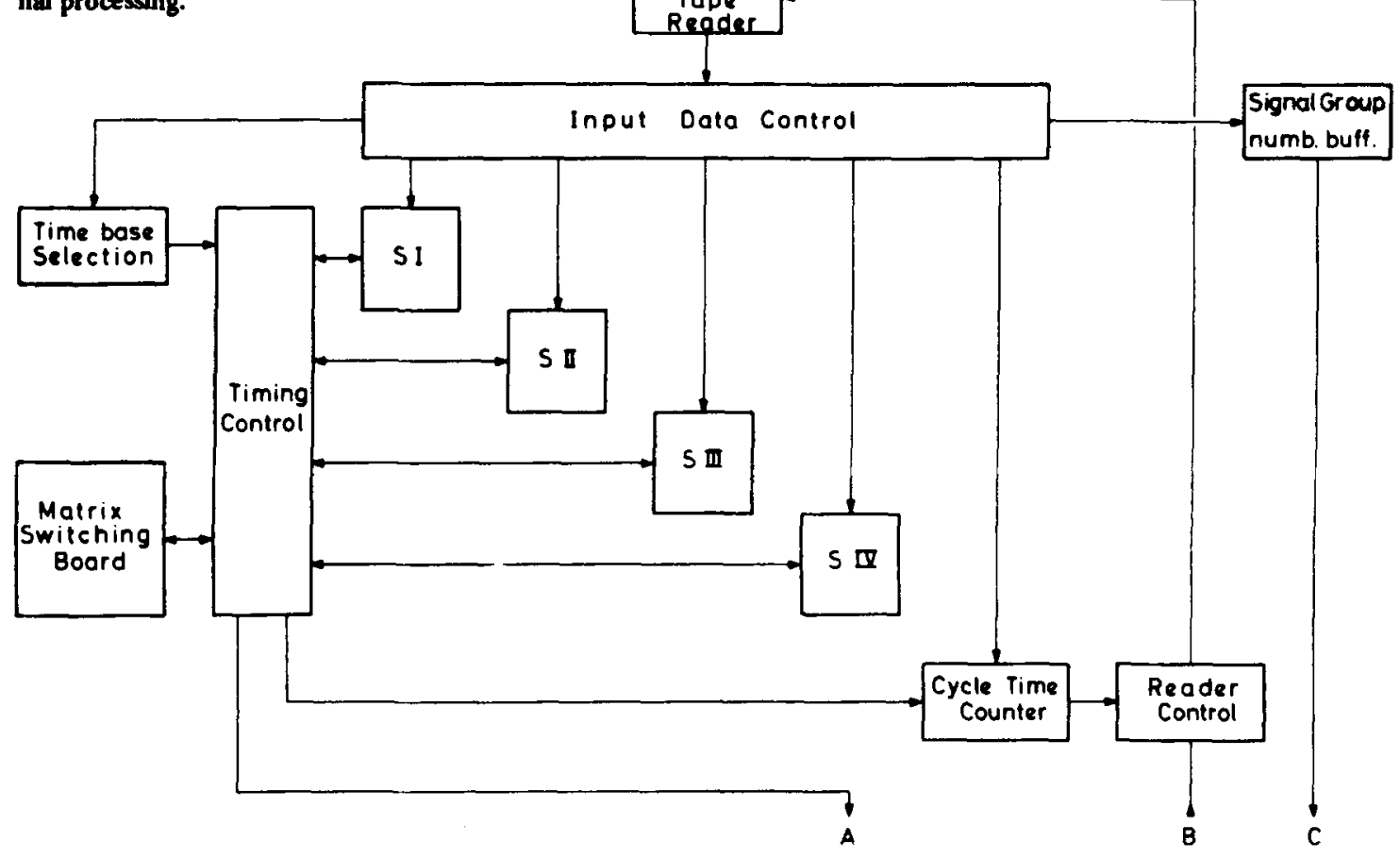




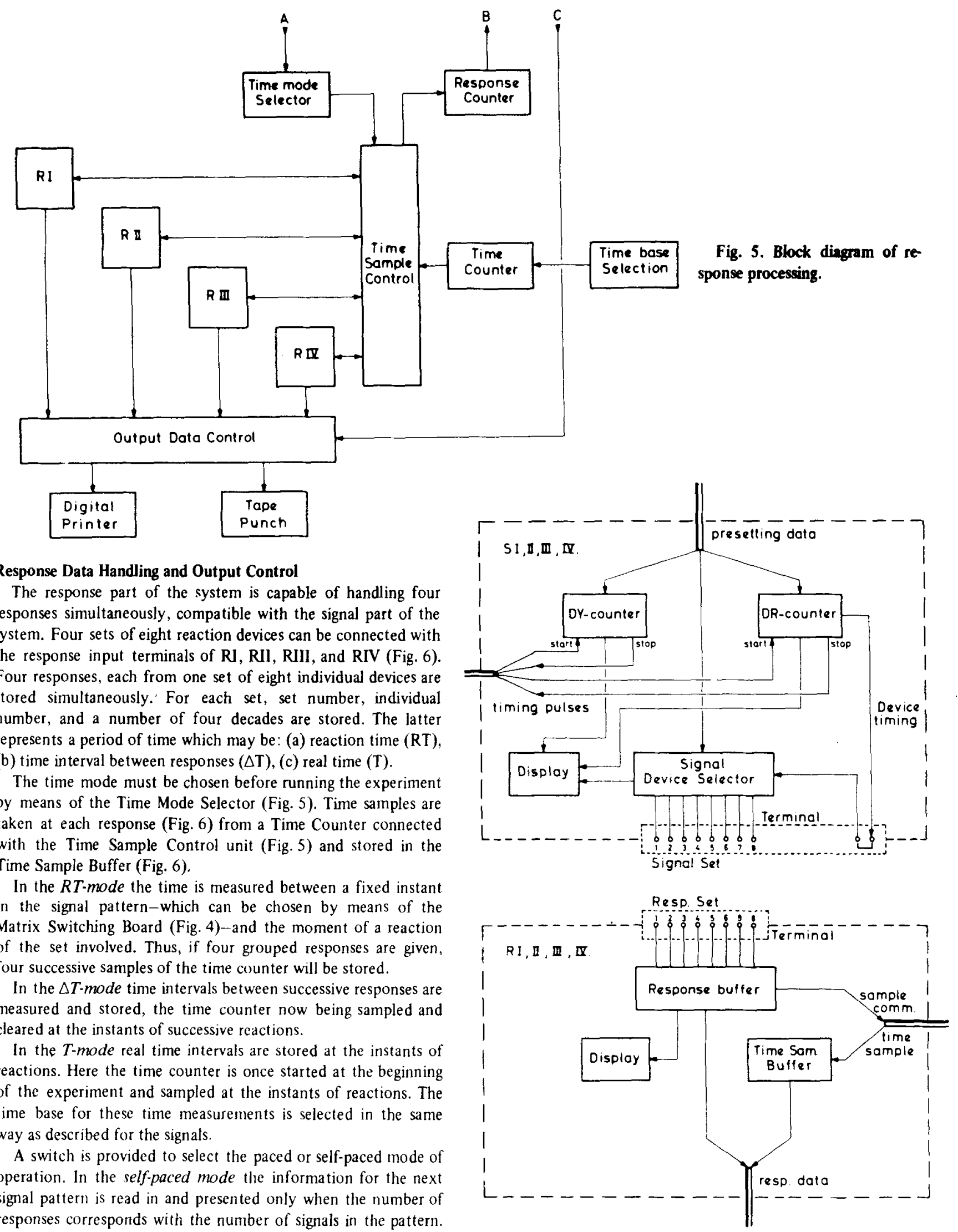

The $E$ can also define the number of responses he wants to wait for before a new signal pattern is presented. This is achieved by setting a switch for the Response Counter (Fig. 5). In this mode the cycle time counter can be used to set the maximal waiting

Fig. 6. Above: Diagram of Signal Sets I, II, MI, and IV of Fig. 4. Below: Diagram of Response Sets I, II, III, and IV of Fig. 5. 
time for all responses to arrive. A new cycle is initialized at the instant the cycle time counter halts. However, if a sufficient number of responses has not yet been given before this counter halts, the new cycle is initialized at the instant of the last response. In the paced mode the signal patterns succeed automatically as described before.

\section{Peripherals for Output}

Two peripherals are available for data output: a Digital Printer and a Tape Punch (Fig. 5). As the system has a storing capacity for data of four responses coming simultaneously, the rate of signal pattern presentation is limited by the maximum speed of the slowest output device. Although printing provides an on-line display of response data, its data feed-out rate is about a quarter of that of the tape punch (e.g., five responses in $1 \mathrm{sec}$ ). Thus, for pilot experiments and trials, responses are printed and punched, or printed only, whereas the printer is switched off for fast and complex experiments.

A signal group number is added to the response data (Fig. 4 and 5) in order to label the momentary signal pattern in the response output data. As well as facilitating interpretation of the printed output, this number is indispensable in the punched output tape.

\section{First Applications}

The first experimental project which made use of PSARP was carried out while the response processing part of the system was only partly finished. So, processing of judgment was possible at that time but not of reaction time. The project concerned a series of experiments on short-term memory for discrete spatial positions. Twenty-six lights, each corresponding with a specific individual from a set in PSARP, were mounted in a 13 by 2 matrix on a board. The lights had been fixed on top of reaction keys, enabling $S$ s to respond by just touching the particular light. In a typical experiment, a variable number of lights flashed successively, preceded by a warning signal and an indication about the number of flashes. Since the total signal capacity of PSARP is 32 , six lights could be used to program the warning signals. An "empty" cycle, i.e., a cycle in which only a cycle time was programmed, controlled the interval between presentation of a trial and the next warning signal.

It is clear that the possibilities of PSARP are only partly used in these experiments (see Sanders, 1968). More intensive use of the possibility to present several signals simultaneously or overlapping is made in experiments on the psychological refractory period. PSARP enables presentation of up to four signals in rapid succession, instead of the traditional series of two signals. All time intervals have an accuracy of 1 msec. This feature makes the apparatus very appropriate for the study of judgment of simultaneity, when signals are presented in succession, but spaced with very small intervals. It is clear from these examples that PSARP can be used for a variety of experiments on human perceptual motor skills, while the experimental task can reach a considerable level of complexity.

\section{REFERENCES}

BERTELSON, P. Central intermittency twenty years later. Quart. J. exp. Psychol., 1966, 18, 153-163.

BROADBENT, D. E., \& GREGORY, M. Stimulus set and response set: The alternation of attention. Quart. J. exp. Psychol., 1964, 16, 309-317.

SANDERS, A. F. Short term memory for spatial positions. Ned. T. voor Psychol, 1968, 28, 1.16.

SANDERS, A. F., \& van BORSELSEN, J. W. Continuing memory and information processing. J. exp. Psy'chol., 1966, 71, 844-848.

\section{Report of a nonnutritive food} substance, palatable to rats

\author{
CHRISTOPHER TAYLOR AND JAMES L. BRUNING, OHIO \\ UNIVERSITY, Athens, Ohio
}

Formulas are presented for two food substances matched in taste and texture characteristics, one nutritive $(4.00 \mathrm{cal} / \mathrm{g}$ ) and the other nonnutritive $(0.00 \mathrm{cal} / \mathrm{g})$. Mean consumption amounts and body weight changes over a five day period are given for both male and female rats. Areas of possible application are suggested for experiments where natural ingestion of bulk is desired.

A nonnutritive food substance (caloric value $=0.0032 \mathrm{cal} / \mathrm{g}$ ) has been devised which rats will eat. Taste and texture characteristics of this nonnutritive substance were matched as closely as possible to those of an adulterated version $(4.00 \mathrm{cal} / \mathrm{g})$ of a high-fat substance $(6.00 \mathrm{cal} / \mathrm{g})$ reported by $\mathrm{Smith}$, Pool, \& Weinberg (1962). The substances have a taste and texture judged by humans to be similar to the standard dough of "refrigerator cookies".

Both substances (with vitamin supplement of $4 \mathrm{~g}$, Nutritional Biochemicals, added to the nutritive substance) have been used as the sole diet for a five day period (Taylor \& Bruning, 1967). All animals were 150 days old; additional growth was minimal. Body weight changes, previously unreported, were as follows: males (mean start weight $=435 \mathrm{~g}$ ) on nonnutritive $(\mathrm{NN})$ substance lost approximately $17 \%$ of their initial body weight and ate an average of $32 \mathrm{~g}$ of $\mathrm{NN}$ substance daily; females (mean start weight = $301 \mathrm{~g}$ ) on NN substance lost approximately $16 \%$ of their initial body weight and ate an average of $42 \mathrm{~g}$ of NN substance daily. In contrast, males (mean start weight $=426 \mathrm{~g}$ ) on nutritive $(\mathrm{N})$ substance gained approximately $4 \%$ of their initial body weight and ate an average of $39 \mathrm{~g}$ of $\mathrm{N}$ substance daily; females (mean start weight $=301 \mathrm{~g}$ ) on $\mathrm{N}$ substance gained approximately $3 \%$ of their initial body weight and ate an average of $28 \mathrm{~g}$ of $\mathrm{N}$ substance daily.

The body weight changes under NN substance schedule compare favorably with body weight changes reported by Bolles (1965) of animals on continuous food deprivation. Bolles found that 270-day-old males after five days deprivation lost $17 \%$ of the body weight of their matched, undeprived controls; that 150 day-old males after five days deprivation lost $18 \%$ of the body weight of their controls; that females (120-days-old) after four days deprivation lost $21 \%$ of the body weight of their controls. It should be noted that rats on NN substance diet drink considerable amounts of water-a factor which may have made weight measurements in the present study somewhat high. The body weight gain under $N$ substance diet in the present study would be expected since the caloric value of this substance $(4.00 \mathrm{cal} / \mathrm{g})$ exceeds the caloric value of laboratory Purina Chow (3.61 cal $/ \mathrm{g}$; Corbit \& Stellar, 1964).

The recipes for the respective substances follow:

Nonnutritive Substance. (approximately $0.0032 \mathrm{cal} / \mathrm{g}$ ) $50 \mathrm{~g}$ methyl cellulose ( 4000 centipoise, Fisher Scientific)

$70 \mathrm{~g}$ "'aseline"

$36 \mathrm{~g}$ calcium carbonate

$28 \mathrm{~g}$ mineral oil (heavy weight)

$4 \mathrm{~g}$ salt mixture (U.S.P. XIV, Nutritional Biochemicals)

$50 \mathrm{ml}$ "Diet-Pepsi" (non-carbonated fountain syrup)

$24 \mathrm{ml}$ tap water 